\title{
ELECCIONES MUNICIPALES, AUTONÓMICAS Y EUROPEAS EN ESPAÑA (13 DE JUNIO DE 1999)
}

Coordinación: CARLOS VIDAL PRADO

Profesor Asociado del Departamento de Derecho Constitucional en la UNED 



\title{
ELECCIONES MUNICIPALES, AUTONÓMICAS Y EUROPEAS EN ESPAÑA (13 DE JUNIO DE 1999)
}

POR

\author{
CARLOS VIDAL PRADO \\ Profesor Asociado del Departamento de Derecho Constitucional \\ en la UNED
}

\section{NOTA INTRODUCTORIA}

El pasado 13 de junio de 1999 se celebraron en España conjuntamente elecciones europeas, municipales y autonómicas. Las dos primeras convocatorias afectaron a todo el territorio nacional. Las elecciones autonómicas se celebraron en todas las Comunidades excepto Cataluña, País Vasco, Galicia y Andalucia. Como es habitual en nuestra Revista, publicamos un comentario sobre esta convocatoria electoral. Ante la imposibilidad de analizar pormenorizadamente todos los resultados', hemos optado por recoger algunos aspectos parciales de los datos, junto con comentarios algo más extensos referidos a las elec-

1 En algunos casos, como en el de las elecciones municipales, resulta complejo acceder a los resultados definitivos, pues no han sido todavía publicados de modo completo en el BOE. La Junta Electoral Central, por Acuerdo de 15 de julio de 1999, ordenó que se procediese a la publicación del resumen de los resultados de las elecciones locales convocadas por Real Decreto 606/1999, de 19 de abril, y celebradas el 13 de junio, según los datos que figuran en las actas de proclamación remitidas por cada una de las Juntas Electorales de Zona. Esta publicación comenzó en el BOE del 27 de julio de 1999 (el Acuerdo aparece en la página 27981 de dicho $B O E)$, pero los fascículos en los que se publican los resultados no han terminado todavía de ver la luz. 
ciones locales en Galicia, a las forales en Navarra y a las europeas a nivel nacional.

\section{CUADROS DE RESULTADOS DE LAS ELECCIONES AUTONÓMICAS DE $1999^{2}$}

\section{Aragón}

\begin{tabular}{|c|c|c|c|}
\hline $\begin{array}{l}\text { Votos emitidos } \\
\text { Participación } \\
\text { Abstención }\end{array}$ & $\begin{array}{r}654.944 \\
65 \% \\
35 \%\end{array}$ & & \\
\hline Candidaturas & Votos & Porcentaje & $\begin{array}{l}\text { Escaños } \\
\text { (Esc. 95) }\end{array}$ \\
\hline PP & 248.781 & 38,26 & $28(27)$ \\
\hline PSOE & 199.620 & 30,70 & $23(19)$ \\
\hline PAR & 86.246 & 13,26 & $10(14)$ \\
\hline $\mathrm{CHA}$ & 72.129 & 11,09 & $5(2)$ \\
\hline IU & 24.805 & 3,8 & $1(5)$ \\
\hline SOS NAT & 3.627 & 0,56 & - \\
\hline PH & 1.101 & 0,17 & - \\
\hline ARTA & 350 & 0,05 & - \\
\hline Votos nulos & 4.668 & & \\
\hline Votos blanco & 13.617 & & \\
\hline Total & 654.944 & - & 67 \\
\hline
\end{tabular}

\section{Canarias}

$\begin{array}{lr}\text { Total de Votos } & 830.352 \\ \text { Votos en Blanco } & 12.558 \\ \text { Votos Nulos } & 4.829\end{array}$

2 Fuente: datos oficiales del Gobierno o Parlamento de cada Comunidad Autónoma publicados en Internet. 
ELECCIONES MUNICIPALES, AUTONÓMICAS Y EUROPEAS EN ESPAÑA...

\begin{tabular}{lccc}
\hline Candidaturas & Votos & Porcentaje & $\begin{array}{c}\text { Escaños } \\
\text { (Esc. 95) }\end{array}$ \\
\hline CC & 306.658 & 36,93 & $24(22)$ \\
PP & 225.316 & 27,14 & $15(18)$ \\
PSOE & 199.503 & 24,03 & $19(16)$ \\
FEDERACIÓN & & & \\
NACIONALISTA CANARIA & 39.947 & 4,81 & 0 \\
IU & 22.768 & 2,74 & 0 \\
LOS VERDES & 12.146 & 1,46 & 0 \\
UC-CDS & 4.442 & 0,54 & 0 \\
AGRUPACIÓN HERRENA & & & \\
INDEPENDIENTE & 2.773 & 0,33 & $2(1)$ \\
PH & 1.346 & 0,16 & 0 \\
ALTERNATIVA MAGA & & & \\
NACIONALISTA & 864 & 0,10 & 0 \\
ALTERNATIVA CANARIA & 806 & 0,10 & 0 \\
TAGOROR PENSIONISTA & & & \\
DE CANARIAS & 692 & 0,08 & 0 \\
PLATAFORMA POPULAR & & & \\
DE FUERTEVENTURA & 533 & 0,06 & 0 \\
\hline
\end{tabular}

\section{Cantabria}

Votos emitidos $\quad 319.947$

Participación $\quad 68,78 \%$

\begin{tabular}{lrcc}
\hline Candidaturas & Votos & Porcentaje & $\begin{array}{c}\text { Escaños } \\
\text { (Esc. 95) }\end{array}$ \\
\hline PP & 134.924 & 42,50 & $19(13)$ \\
PSOE & 105.004 & 33,08 & $14(10)$ \\
PRC & 42.896 & 13,51 & $6(6)$ \\
IU & 11.707 & 3,69 & $0(3)$ \\
UPCA & 9.743 & 3,07 & $0(7)$ \\
\hline
\end{tabular}




\section{Castilla y León}

Censo

2.110 .422

Participación

1.464 .809

Abstención

645.613

$69,41 \%$

Votos en blanco

38.990

$30,59 \%$

Votos nulos

13.956

$2,66 \%$

$0,95 \%$

\begin{tabular}{lrcc}
\hline Candidaturas & Votos & Porcentaje & $\begin{array}{c}\text { Escaños } \\
\text { (Esc. 95) }\end{array}$ \\
\hline PP & 733.557 & 50,56 & $48(50)$ \\
PSOE & 478.060 & 32,95 & $30(27)$ \\
UPL & 53.791 & 3,71 & $3(2)$ \\
IU & 78.933 & 5,44 & $1(5)$ \\
TC-PNC & 20.063 & 1,38 & $1(0)$ \\
URCL & 10.985 & 0,76 & 0 \\
UC-CDS & 10.171 & 0,70 & 0 \\
CI & 6.837 & 0,47 & 0 \\
PB & 3.644 & 0,25 & 0 \\
PADE & 3.165 & 0,22 & 0 \\
PREPAL & 2.912 & 0,20 & 0 \\
PH & 2.232 & 0,15 & 0 \\
USI & 1.779 & 0,12 & 0 \\
UPZ & 1.484 & 0,10 & 0 \\
LV-GV & 1.367 & 0,09 & 0 \\
FE-JONS & 997 & 0,07 & 0 \\
LV & 777 & 0,05 & 0 \\
PAE-I & 560 & 0,04 & 0 \\
PCPE & 283 & 0,02 & 0 \\
PANCAL & 276 & 0,02 & 0 \\
\hline
\end{tabular}

\section{La Rioja}

Censo

Participación

Votos nulos

Votos en blanco

Votos válidos
229.569

157.623

1.377

3.535

156.246 
ELECCIONES MUNICIPALES, AUTONÓMICAS Y EUROPEAS EN ESPAÑA...

\begin{tabular}{lrcc}
\hline Candidaturas & Votos & Porcentaje & $\begin{array}{c}\text { Escaños } \\
\text { (Esc. 95) }\end{array}$ \\
\hline PP & 88.088 & 51,26 & $18(17)$ \\
PSOE & 55.126 & 35,28 & $13(12)$ \\
PR & 9.004 & 5,76 & $2(2)$ \\
IU & 6.104 & 3,91 & $0(2)$ \\
Verdes & 1.971 & 1,26 & 0 \\
MASH & 418 & 0,27 & 0 \\
\hline
\end{tabular}

\section{Madrid}

Participación $\quad 60,88 \%$

\begin{tabular}{lrcc}
\hline Candidaturas & Votos & Porcentaje & $\begin{array}{c}\text { Escaños } \\
\text { (Esc. 95) }\end{array}$ \\
\hline PP & 1.324 .596 & 51,07 & $55(54)$ \\
PSOE-Progresistas & 944.819 & 36,43 & $39(32)$ \\
IU & 199.488 & 7,69 & $8(17)$ \\
Otros & 70.251 & 2,71 & 0 \\
\hline
\end{tabular}

\section{Comunidad Valenciana}

Censo escrutado $\quad 3.339 .798 \quad 100,00 \%$

\begin{tabular}{lrcc}
\hline Datos totales & Votos 99 & Porcentaje 99 & Porcentaje 95 \\
\hline Número de votantes & 2.276 .461 & 68,16 & 76,04 \\
Abstención & 1.063 .337 & 31,84 & 23,96 \\
Votos nulos & 13.686 & 0,60 & 0,55 \\
Votos en blanco & 35.562 & 1,56 & 1,04 \\
Votos válidos & 2.262 .775 & & \\
Votos a candidaturas & 2.227 .213 & 97,84 & 98,41 \\
\hline
\end{tabular}


CARLOS VIDAL PRADO

\begin{tabular}{lrrc}
\hline Candidaturas & Votos & Porcentaje & $\begin{array}{c}\text { Escaños } \\
\text { (Esc. 95) }\end{array}$ \\
\hline PP & 1.083 .306 & 48,64 & $49(42)$ \\
PSOE-progresistas & 767.442 & 34,46 & $35(32)$ \\
EUPV & 136.836 & 6,14 & $5(10)$ \\
U.V. & 105.987 & 4,76 & $0(5)$ \\
BLOC-VERDS & 102.673 & 4,61 & 0 \\
A.C.V. & 6.107 & 0,27 & 0 \\
C.L. & 4.807 & 0,22 & 0 \\
L.V.E & 4.077 & 0,18 & 0 \\
FE-JONS & 2.860 & 0,13 & 0 \\
UC-CDS & 2.722 & 0,12 & 0 \\
I.I. & 2.521 & 0,11 & 0 \\
PH & 2.236 & 0,10 & 0 \\
E.N.V. & 2.187 & 0,10 & 0 \\
IRF-PRF & 1.607 & 0,07 & 0 \\
O.I.V. & 1.287 & 0,06 & 0 \\
L.A.E. & 558 & 0,03 & 0 \\
\hline
\end{tabular}

NÚMERO DE ESCAÑOS OBTENIDOS EN OTRAS COMUNIDADES ${ }^{3}$ (entre paréntesis, escaños y porcentajes en 1995)

\begin{tabular}{|c|c|c|c|c|c|c|c|c|}
\hline Comunidad & $P p$ & $\%$ & PSOE & $\%$ & IU & $\%$ & Otros & $\%$ \\
\hline Murcia & $27(26)$ & $52,9(52,2)$ & $17(15)$ & $35,8(31,8)$ & $1(4)$ & $6,9(12,4)$ & & \\
\hline Extremadura & $28(27)$ & $40,1(39,5)$ & $34(31)$ & $48,4(43,8)$ & $3(6)$ & $6(10,5)$ & EU 0 (1) & \\
\hline Castilla La Mancha & $21(22)$ & $40,4(44,3)$ & $26(24)$ & $53,3(45,7)$ & $0(1)$ & & & \\
\hline Baleares & $28(30)$ & $43,9(44,7)$ & $13(16)$ & $22(23)$ & $3(3)$ & $5,4(6,6)$ & $\begin{array}{l}\text { PSM } 5(6) \\
\text { UM } 3(2) \\
\text { Pacte } 6(0) \\
\text { AlPF } 1(1)\end{array}$ & $\begin{array}{l}11,8(12,2) \\
7,3(5,3) \\
4,5(0) \\
0,4(0,7)\end{array}$ \\
\hline Asturias & $15(21)$ & $32,3(41,9)$ & $24(17)$ & $45,9(33,8)$ & $3(6)$ & $9(16,4)$ & $\begin{array}{l}\text { URAS } 3(0) \\
\text { PAS } 0(1)\end{array}$ & \\
\hline
\end{tabular}

3 Sobre estas Comunidades no se dispone de información oficial en Internet. 
ELECCIONES MUNICIPALES

Distribución de mayorías por partidos en los ayuntamientos

\begin{tabular}{lrrr}
\hline Partido & Absoluta & Relativa & Empate \\
\hline PP & 3.175 & 438 & 6 \\
PSOE & 2.045 & 460 & 5 \\
CIU & 515 & 87 & 1 \\
IU & 77 & 45 & 1 \\
PAR & 152 & 26 & 1 \\
EH & 41 & 22 & \\
ERC-AM & 23 & 14 & \\
EAJ-PNV & 70 & 34 & 1 \\
BNG & 6 & 1 & \\
PA & 18 & 15 & \\
CC & 24 & 7 & \\
EAJ-PNV/EA & 21 & 19 & \\
UC-CDS & 19 & 9 & \\
UV & 4 & 5 & 1 \\
PRC & 13 & 7 & \\
BLOC-VERDS & 4 & & \\
IC-V-EPM & 6 & 3 & \\
EA & 9 & 9 & \\
FIC & 14 & 2 & \\
UPL & 4 & 3 & \\
URCL & 12 & 4 & \\
PSM-EN & 5 & & \\
GIL & 2 & 6 & \\
URAS & 3 & 5 & \\
CHA & 7 & & \\
UM & 2 & 1 & \\
PR & 10 & & \\
PREX-CREX & 3 & 1 & \\
EU & 3 & 1 & \\
RESTO & 390 & 120 & \\
\hline
\end{tabular}




\title{
ELECCIONES LOCALES Y EUROPEAS EN GALICIA
}

\author{
ÁLVARO XOSÉ LÓPEZ MIRA
}

Profesor Titular de Ciencia Política

Universidad de Vigo

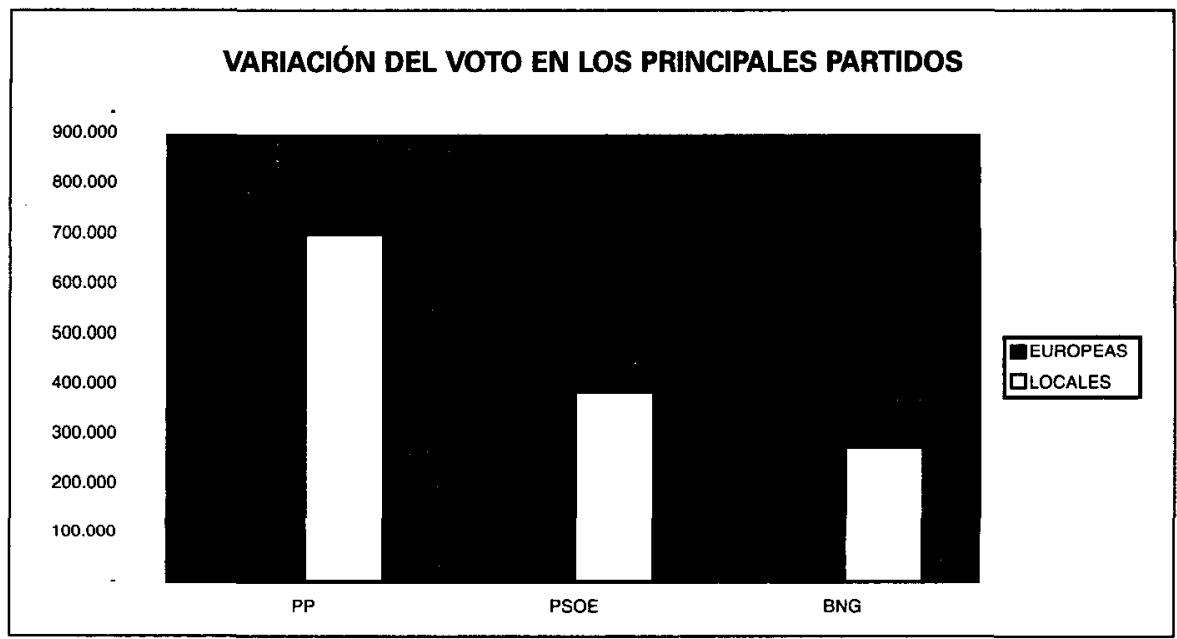

Una situación política compleja y, por lo tanto, de gran interés para el estudioso, como lo es la que se da en la Comunidad Autónoma de Galicia, no puede valorarse con la simpleza utilizada por ciertos medios de comunicación y algunos líderes políticos, en coincidente armonía, de los dos mayores partidos españoles. El reduccionismo de la amenaza de un tercer foco de enfebrecido nacionalismo parece exclusivamente ideado para generar titulares escandalosos (y ojalá esta fuese la peor de las intenciones que cupiera vislumbrar). La reciente pregunta de una periodista en un curso de verano al profesor Beiras acerca de si con el Bloque Nacionalista Galego (BNG) había llegado el apocalipsis (sic), es algo que ni en el fragor de la campaña electoral se les ha ocurrido insinuar a sus más rancios y/o recalcitrantes rivales políticos.

A riesgo de simplificar también nosotros, convendría indicar cómo parece que el panorama que comienza a dibujarse en Galicia es el de la pérdida del monopolio político del Partido Popular en esta Comunidad Autónoma que rige con pocos altibajos desde 1989: tres mayorías absolutas en la Cámara autonómica, dos tercios de los munici- 
pios (en 1995 casi los dos tercios de la población) y las cuatro Diputaciones, excelentes cosechas de escaños en las elecciones supragallegas ${ }^{4}$ y un incremento constante de la participación desde la entrada de Manuel Fraga en la escena política de esta nacionalidad histórica, caminando en paralelo con el aumento de la cifra de votos de su partido.

Pues bien, como han señalado los profesores Arias y Cancio ${ }^{5}$ las distancias (en número de votos, que no de escaños) entre la opción política dominante y sus competidores alternativos se han estrechado desde 1977 e incluso han cambiado de signo en las generales de 1996. En ningún momento desde la transición el electorado gallego se mantuvo políticamente estático, pero en 1999 los partidos parlamentarios de la oposición gallega suman tantos sufragios en las elecciones locales como el Partido Popular 6 .

Otra tendencia, ya observable con anterioridad, que se afianza en esta última convocatoria electoral es la emisión de voto dual por parte de los gallegos en función del tipo de elección. PP y BNG superan en las europeas ampliamente los votos que obtienen en las locales ( 31.000 y 41.000, respectivamente), al contrario que el PSOE (47.000 sufragios menos en las europeas). Así, en el municipio de A Coruña la lista local de este partido obtuvo 26.000 votos más que su homónima europea y 5.000 más en el de Santiago de Compostela. Sin embargo, la lista popular coruñesa consiguió 15.000 sufragios menos que la europea y 7.000 menos la viguesa.

Una tercera inferencia (que también refuerza el dato citado) es el retorno del PSOE a su tradicional papel de segunda fuerza política en la Comunidad debido al tipo de elección, ya que los carismáticos alcaldes socialistas lograron frenar las constantes caídas de voto de su partido en anteriores comicios de otra índole (generales o, especialmente, autonómicos). De todas maneras, la auténtica medida de este parcial éxito deberá darse en las próximas elecciones generales, proclives a generar situaciones de bipartidismo (en el Senado es pleno) entre PP y PSOE; si el BNG también sobrepasa a los socialistas en este tipo de

4 Cierto que nunca superando los resultados de UCD en 1977 y 1979.

5 Vid. Pedro Arias Veira y Miguel Cancio Álvarez, Las elecciones en Galicia. De la hegemonía centrista a la competencia tripartidista. 1977-1997, Santiago de Compostela, Tórculo Edicións, 1999.

6 Habría que tener en cuenta la presencia de numerosas candidaturas independientes en este tipo de comicios, la mayoría de ellas de centro-derecha, $o$ incluso escindidos del propio Partido Popular. 
convocatoria podría augurarse una continuidad del descenso pese a conservar algunos feudos locales ${ }^{7}$, como A Coruña ${ }^{8}$.

Pero quizás la conclusión más interesante que se puede extraer de estos comicios locales en Galicia sea la amargura de la nueva victoria del PP, muy clara a todos los niveles (número de votos, porcentaje superior al $45 \%$ y por encima del $53 \%$ en las provincias de Lugo y Ourense, 216 Ayuntamientos de los 315 , con mayoría absoluta y pleno en las cuatro Diputaciones). Pero en las siete grandes ciudades gallegas ese porcentaje global disminuye en siete puntos y medio, obteniendo únicamente la alcaldía de Ourense, lo que implica que la mayoría de la población gallega estará gobernada por los principales partidos de la oposición: BNG (Vigo, Pontevedra y Ferrol) y PSOE (A Coruña, Santiago de Compostela y Lugo).

En este sentido, el incremento del voto en las grandes urbes del BNG y la relativa estabilidad del PSOE $^{9}$ podría marcar un punto de inflexión en la dinámica política gallega. No se debe olvidar que la victoria electoral de Fraga en 1989 (y las sucesivas) se cimentó, sobre todo, en el voto urbano, ganando en las siete ciudades y en las villas más importantes. Si este cambio observable en la tendencia de voto de este electorado se ve avalado por unos gobiernos progresistas sólidos y eficaces, probablemente estemos asistiendo al primer acto del fin de la hegemonia del PP en Galicia ${ }^{10}$.

Concretamente, la provincia de Pontevedra, con las alcaldías del motor económico y ciudad más importante de Galicia, Vigo, para el BNG, así como la capital provincial (donde empató con el PP en número de concejales, duplicando ambos al PSOE), va a influir también en la política interna de los populares gallegos especialmente la baronía territorial de José Cuiña.

En relación con los pactos entre las fuerzas progresistas (PSOEBNG) resulta difícilmente comprensible la completa falta de documen-

7 Téngase en cuenta que el Presidente de la Xunta ha insinuado la posibilidad de adelantar las elecciones autonómicas (previstas para el 2001) para hacerlas coincidir con las generales.

8 Cuanto mayores son los éxitos de Francisco Vázquez en su ciudad, peores resultados obtiene su partido en el conjunto de Galicia.

9 Habría que añadir también los gobiernos de la mayor parte de las villas medias (más de veinte mil habitantes), como Narón, Oleiros, Vilagarcía de Arousa, Marín o Redondela.

10 Aunque tanto poder acumulado y su importante apoyo clientelar y social (más de $\mathbf{1 0 0 . 0 0 0}$ afiliados) no se derrumbará con un solo embate ni en breve plazo. 
tación de los medios informativos de la capital del Estado. Tras las elecciones locales de 1995 se suscribieron idénticos acuerdos" entre los mismos partidos con valoraciones, en general, positivas por ambas partes, sin desatar ningún trueno mediático, sino con absoluta naturalidad $y$, por supuesto, respeto de su rival, el PP gallego ${ }^{12}$, siendo en aquel entonces Secretario General de los socialistas gallegos el ahora crítico con ellos Francisco Vázquez, quien estuvo muy cerca de perder la mayoría absoluta en dichos comicios $y$, por tanto, de precisar el apoyo de los concejales nacionalistas. Todavía más, negar credibilidad democrática a dichos pactos por parte de quien necesita el apoyo parlamentario de tres partidos nacionalistas de distinto talante, implica no ya escasa legitimación en quien tal afirma, sino un gran desconocimiento del escenario político de Galicia.

De todas formas, habría que añadir que en las ciudades donde el BNG fue partido más votado que el PSOE, éste no entró a formar parte de los gobiernos municipales, apoyando sólo la investidura de Alcaldes nacionalistas ${ }^{13}$. No ha sucedido asi en las urbes donde los socialistas recibieron más votos (Santiago de Compostela y Lugo), ya que en ellas los concejales del BNG asumieron responsabilidades de gobierno, lo que muestra una diferencia de talante y también una mayor seriedad de objetivos políticos generales.

Por último, tendríamos que valorar como indeseable la demonización que se está efectuando del nacionalismo político gallego a medida que crecen sus expectativas electorales $y$, consiguientemente, su peso específico en el juego democrático, como si fuera indeseable su homologación política. Esta coalición electoral es, en la actualidad, la quinta fuerza política más votada en España ${ }^{14}$; la extrapolación de los últimos resultados electorales le otorgaria grupo parlamentario en el Congreso con, al menos, seis diputados. $Y$ aunque en estos momentos no sea más que mera especulación política, un previsible empate téc-

n Lógicos y hasta inevitables en una situación de partido dominante como la que se vive en Galicia.

12 Es muy frecuente que en el Parlamento autonómico los dos partidos de la oposición apoyen conjuntamente sus respectivas propuestas contra la mayoría.

13 Posteriormente, en las ciudades de Vigo y Ferrol, los concejales socialistas han hecho perder varias votaciones a sus hipotéticos socios, posicionándose erráticamente en ellas con el Partido Popular.

14 Simplemente como anécdota cabría señalar que, en los comicios europeos, obtuvo votos en todas las provincias españolas, Ceuta y Melilla incluidas, siendo Madrid la que mayor número de sufragios aportó (más de 4.500) tras las cuatro gallegas. 
nico entre PP y PSOE en esta Cámara, le podría facilitar a este último partido un aliado natural para la formación de mayorías, dado que, en la actualidad, los nacionalistas gallegos (aparte de autoposicionarse en la izquierda) no pueden permitirse la boutade de pactar con el PP en Madrid, mientras este partido siga ostentando tanto poder en la Comunidad gallega. 


\title{
ELECCIONES AUTONOMICAS DEL 13-J 1999 EN NAVARRA
}

\author{
ASUNCIÓN DE LA IGLESIA CHAMARRO \\ Profesora de Derecho Constitucional \\ Universidad de Navarra
}

Se dibuja en la Comunidad Foral un mapa electoral distinto del común a la mayoría de las Comunidades Autónomas españolas. Ello se debe principalmente a la presencia de dos tendencias políticas bien definidas: de un lado, un regionalismo navarro dominante, y de otro, un nacionalismo vasco apoyado con más o menos intensidad en sus distintas manifestaciones (Euskal Herritarrok, Partido Nacionalista Vasco y Eusko Alkartasuna).

Buena muestra de lo peculiar del mapa electoral navarro es que el actual gobierno regionalista de Unión del Pueblo Navarro (Partido Popular en Navarra) $)^{15}$ se apoya en un pacto de investidura con el partido socialista (PSN-PSOE). Resulta asi que la rivalidad de estos dos grupos en el panorama nacional es hoy en Navarra pacto de gobierno.

En cuanto a los aspectos instrumentales, baste decir que los cincuenta parlamentarios forales son elegidos por sufragio universal, libre, igual, directo y secreto en una circunscripción única (la Comunidad Foral) ${ }^{16}$ y siguiendo para la atribución de escaños la fórmula proporcional D'Hondt. Es la Ley foral 16/1986 de 17 de noviembre la que regula las elecciones al Parlamento de Navarra.

Antes de presentar los resultados y analizar participación y distribución del voto entre las fuerzas políticas apuntamos unas brevísimas notas sobre la campaña electoral y las circunstancias particulares de las principales fuerzas políticas contendientes.

\section{La campaña electoral}

Al hablar de las autonómicas de 1999 resulta insoslayable la referencia al clima derivado de la tregua de ETA desde septiembre de

15 Unión del Pueblo Navarro, creado en 1979 para defender la identidad navarra y consolidar el régimen foral, se une en marzo de 1991 al Partido Popular, compareciendo así el primero como único partido de centro-derecha en Navarra.

16 Antes de la Ley Orgánica de Reintegración y Amejoramiento del Fuero (LORAFNA), LO 13/1982 de 10 de agosto eran seis las circunscripciones y setenta los parlamentarios forales. 
1998 y al pacto de Estella. Veremos cómo estos presupuestos han influido en el comportamiento de parte del electorado.

Hablando ya de los grupos políticos en particular, el regionalista de centro-derecha Unión del Pueblo Navarro (UPN), comparece ante los electores como partido en el Gobierno, con un mensaje de continuidad y subrayando los éxitos nacionales y forales en el plano de la economía. Repite como candidato a la Presidencia Miguel Sanz, quien ya accedió a la misma en octubre de 1996 tras la dimisión del Presidente socialista Javier Otano Cid por el escándalo de las cuentas en Suiza ${ }^{17}$.

Más novedades presenta el Partido Socialista de Navarra (PSN), que se enfrenta a las elecciones con una imagen renovada, intentando borrar de la memoria colectiva los episodios judiciales de los dos expresidentes socialistas del Gobierno de Navarra, Gabriel Urralburu y Javier Otano y remontar los pésimos resultados de 1995. El procedimiento de primarias da vía a la candidatura de Juan José Lizarbe quien, tras un año al frente del PSN, es la cara nueva en los carteles socialistas.

Favorece especialmente el marco de la tregua a la coalición nacionalista vasca Euskal Herritarrok (EH) que aglutina a Herri Batasuna y Batzarre. Este grupo político de izquierda nacionalista ha optado en esta ocasión por hacer una campaña más sosegada, dirigida a la captación de votos en Navarra, recurriendo a los símbolos de la tierra como las cadenas o el monumento a los Fueros. Con esta política EH ha querido transmitir que es Navarra quien debe decidir su futuro. Por otra parte, no han faltado gestos clásicos de la coalición Herri Batasuna como la inclusión de presos en las listas o los vítores a la organización ETA en los mítines, $y$, por supuesto la reivindicación de la autodeterminación. Como curiosidad, es de señalar el anuncio hecho por el candidato de EH a la presidencia del Gobierno Foral -Pernando Barrena- según el cual Pamplona sería la capital del Estado Federal Vasco.

Expectante ante el apoyo real del electorado comparece en estas elecciones Convergencia de Demócratas Navarros (CDN). Son las seguntas forales para este grupo escindido en 1995 de Unión del Pueblo Navarro y formado en torno al ex-Presidente regionalista Juan Cruz Alli.

Poco espacio se prevé para lizquierda Unida (IU) que, tras su desconcertante actuación en el pacto de Estella, y sumida en un mal mo-

17 Otano accedió a la Presidencia del Gobierno de Navarra tras las elecciones de 1995 que alumbraron un gobierno de coalición: Partido Socialista de Navarra (PSN-PSOE), Convergencia de Demócratas Navarros (CDN) y Eusko-Alkartasuna (EA). 
mento a nivel nacional, lucha por un electorado de izquierda que en el fragmentado mapa electoral navarro tiene otros reclamos más fuertes.

Por último, en 1999, y ante el previsible crecimiento de Euskal Herritarrok, los nacionalistas vascos moderados de Eusko Alkartasuna (EA) y Partido Nacionalista Vasco (PNV) unen sus fuerzas y presentan en estas elecciones una candidatura conjunta.

Los primeros sondeos apuntan ya la victoria de UPN -sin llegar en ningún caso a la mayoría absoluta-, el mantenimiento del PSN y el fuerte ascenso de $\mathrm{EH}^{18}$.

\section{Cuadro de resultados}

A continuación se exponen los resultados en una tabla comparativa que nos muestra la variación del voto con respecto a las autonómicas de $1995^{19}$.

\begin{tabular}{lrrrrrr}
\hline & 1999 & 1995 & $\% \mathbf{1 9 9 9}$ & $\% 1995$ & Var. & \\
& $\mathbf{2 0}$ & vot. & Var. \% \\
\hline UPN & $\mathbf{1 2 5 . 4 9 7}$ & 91.167 & $\mathbf{4 2 , 3 5 \%}$ & $31,35 \%$ & +34.330 & +11 \\
PSN-PSOE & $\mathbf{6 1 . 5 3 1}$ & 62.021 & $\mathbf{2 0 , 6 1 \%}$ & $20,87 \%$ & -490 & $-0,26$ \\
EH (HB) & $\mathbf{4 7 . 2 7 1}$ & 27.404 & $\mathbf{1 6 , 0 5 \%}$ & $9,22 \%$ & +19.867 & $+6,83$ \\
IU & $\mathbf{2 0 . 8 7 9}$ & 27.773 & $\mathbf{7 , 0 8} \%$ & $\mathbf{9 , 3 5 \%}$ & -6.894 & $-2,27$ \\
CDN & $\mathbf{2 0 . 8 2 1}$ & 55.153 & $\mathbf{7 , 0 7} \%$ & $18,56 \%$ & -34.261 & $-11,49$ \\
EA-PNV & $\mathbf{1 6 . 5 1 2}$ & 13.568 & $\mathbf{5 , 5 9} \%$ & $4,57 \%$ & +2.944 & $+1,02$ \\
\hline
\end{tabular}

18 El domingo 28 de marzo se publica en el Diario de Navarra un sondeo preelectoral del CIES realizado sobre una muestra de 1205 entrevistas personales en los domicilios en el que se adelanta la siguiente intención directa de voto al Parlamento Foral:

Unión del Pueblo Navarro (UPN): 24,7\%

Partido Socialista de Navarra (PSN): $12,9 \%$

Euskal Herritarrok (EH): $9 \%$

Izquierda Unida (IU): 6,1\%

Convergencia de Demócratas Navarros (CDN): 4,7\%

Eusko Alkartasuna-Partido Nacionalista Vasco (EA-PNV): 4,2\%

Otros: $0,9 \%$

No votarán: $8,9 \%$

Votos blancos: $2,1 \%$

No sabe-no contesta: $26,5 \%$

19 Tabla elaborada con los datos ofrecidos por el Gabinete de Prensa del Gobierno foral.

20 Variación.

21 Votos obtenidos por EA en solitario. 


\section{Análisis de los resultados}

\subsection{La participación}

Sobre un censo de 461.729 ciudadanos, casi veinticinco mil más que en las pasadas elecciones al Parlamento foral, la participación fue del $66,25 \%$. Estamos ante la más baja en unas elecciones de esta naturaleza. Esta tendencia descendente se inicia en 1991 y continúa en 1995 y 1999. Las cifras más bajas de participación se dieron en Pamplona con sólo un $60 \%$ de votantes.

A pesar de este dato es de señalar que Navarra ha presentado siempre una alta participación si se la compara con el resto de las Comunidades Autónomas ${ }^{22}$. En la siguiente tabla se puede apreciar la evolución del censo y de la participación en Navarra en las elecciones al Parlamento foral ${ }^{23}$ :

\begin{tabular}{lcccc}
\hline & Censados & Votantes & \% Participación & $\%$ Abstención \\
\hline 1979 & 365.080 & 258.331 & $70,76 \%$ & $29,24 \%$ \\
1983 & 379.692 & 269.050 & $70,86 \%$ & $29,14 \%$ \\
1987 & 393.326 & 286.263 & $72,78 \%$ & $27,22 \%$ \\
1991 & 414.922 & 276.795 & $66,71 \%$ & $33,29 \%$ \\
1995 & 437.776 & 299.570 & $68,43 \%$ & $31,57 \%$ \\
1999 & 461.729 & 305.896 & $66,25 \%$ & $33,75 \%$ \\
\hline
\end{tabular}

3.2. Valoración general de la distribución del voto entre las fuerzas políticas

Como puede observarse en el cuadro de resultados, en los comicios de 1999 se produce un fuerte crecimiento de Unión del Pueblo Navarro y Euskal Herritarrok, se mantienen los socialistas y sufre un descalabro electoral el grupo escindido en 1995 de UPN, Convergencia de Demócratas Navarros.

22 Cfr. Alcantara, M. y Martínez, A. (ed.), Las elecciones autonómicas en España, 1980-1997, ClS, Madrid, 1998, p. 390.

23 Tabla elaborada sobre los datos ofrecidos por el Gabinete de Prensa del Gobierno de Navarra. 
UPN es la primera fuerza política con mucha ventaja sobre las demás. Alcanza los mejores resultados de su historia, superando su techo de 20 escaños. Queda asi muy cerca de la mayoría absoluta. Protagoniza el grupo regionalista la mayor subida porcentual (sube 11 puntos y alcanza el $42,35 \%$ de los votos) y en votos totales (34.330 votos más). El aumento se debe en su mayor parte a la recuperación de los votantes que en 1995 apoyaron al grupo secesionado Convergencia de Demócratas Navarros, que pierde 34.261 votos. Este grupo, alimentado en 1995 por el tirón de la ruptura con UPN del Presidente Juan Cruz Alli, ha sufrido en las forales de 1999 un auténtico descalabro, pasando de 10 a 3 escaños. Experimenta asi el mayor retroceso de los grupos políticos contendientes.

También es muy notable el crecimiento de Euskal Herritarrok. Favorecida por la tregua, Lizarra y la nueva imagen de la coalición que ha sustituido a Herri Batasuna ha sumado en Navarra casi veinte mil votos más. Por su parte, la coalición de Eusko Alkastasuna y Partido Nacionalista Vasco ha permitido llegar hasta el tercer escaño en el Parlamento foral. En total, los grupos nacionalistas vascos suman hoy en Navarra un $21,64 \%$ de los votos; 7,85 puntos más que en las pasadas forales.

Los socialistas del PSN no consiguen aumentar los 11 escaños de las elecciones de 1995, donde bajaron desde los 19 conseguidos en 1991. Muestran estos resultados que el PSN-PSOE no consigue recuperarse del daño de los escándalos e irregularidades.

En Navarra, Izquierda Unida ha corrido parecida suerte que en el resto del territorio nacional. Pierde más de siete mil votos, que le suponen dos escaños menos. Sin embargo, es la cuarta fuerza política de Navarra, por delante de los Convergentes y los nacionalistas vascos moderados.

\section{La composición del Parlamento Foral}

Seis son las fuerzas políticas con representación que dan lugar a un Parlamento fragmentado, donde ningún partido logra mayoría absoluta. Por tendencias ideológicas tenemos que existe una relación de 39 a 11 entre los partidos navarros y de implantación nacional y los nacionalistas vascos. En cuanto a la distribución entre izquierdas y derechas, el navarro es un Parlamento bastante equilibrado: en el centro derecha 22 escaños de UPN y en la izquierda hay que sumar 11 del PSN, 8 de $\mathrm{EH}$ y 3 de IU (22 en total). Los seis restantes corresponden a los nacionalistas vascos de EA y PNV y a los convergentes. 
Ya hemos señalado que aunque el partido mayoritario lo es a mucha distancia de los demás, no alcanza la mayoría absoluta, siendo necesarias las alianzas para formar gobierno estable. Descartada la alianza entre Unión del Pueblo Navarro y los ex-regionalistas de Convergencia de Demócratas Navarros, que por otra parte tampoco da la mayoría absoluta $(22+3)$, se alcanza un pacto de investidura entre regionalistas y socialistas $(22+11)$ que da la presidencia a los primeros. Con este pacto Euskal Herritarrok se ha convertido en la primera fuerza de la oposición.

\begin{tabular}{|c|c|c|}
\hline & Escaños & Variación-1995 \\
\hline UPN & 22 & +5 \\
\hline PSN-PSOE & 11 & 0 \\
\hline $\mathrm{EH}(\mathrm{HB})$ & 8 & +3 \\
\hline CDN & 3 & -7 \\
\hline IU & 3 & -2 \\
\hline PNV-EA ${ }^{24}$ & 3 & +1 \\
\hline
\end{tabular}

Fuentes: Diario de Navarra, especial elecciones.

Datos ofrecidos por el Gabinete de Prensa del Gobierno de Navarra.

24 En estos comicios concurren en una misma candidatura. En las forales de 1995 el PNV no obtuvo representación en el Parlamento de Navarra, mientras Eusko-Alkartasuna obtuvo 2. 


\section{ELECCIONES EUROPEAS}

\section{CRISTINA RODRIGGUEZ COARASA}

\section{Profesora de Derecho Constitucional}

A continuación se muestran los resultados de las elecciones europeas, con un cuadro comparativo de las últimas elecciones celebradas el 13 de junio de 1999 y las anteriores del año 1994. Además, se incluye un cuadro con la actual composición del Parlamento europeo, así como los datos estadísticos referidos al censo electoral y al número total de votos tanto a nivel nacional como europeo.

ESPAÑA

\begin{tabular}{lcc}
\hline & ELECCIONES 1999 & ELECCIONES 1994 \\
\hline Censo & 33.841 .211 & 31.558 .726 \\
Abstenciones & 12.507 .086 & 12.894 .671 \\
Total votantes & 21.334 .125 & 18.664 .055 \\
Nulos & 168.682 & 85.640 \\
Válidos & 21.164 .836 & 18.578 .415 \\
Blancos & 357.567 & 213.621 \\
Participación & $64,34 \%$ & $59,14 \%$ \\
\hline
\end{tabular}

RESULTADOS ELECTORALES

1999

\begin{tabular}{lrrrl}
\hline Grupo Político 99 & N. ${ }^{\circ}$ de Votos & \multicolumn{1}{c}{$\%$} & Escaños & Grupo \\
\hline PP & 8.410 .790 & 40,42 & 27 & PPE (27) \\
PSOE-PROGRESISTAS & 7.476 .549 & 35,93 & 24 & PSE (24) \\
IU-EUIA & 1.221 .440 & 5,87 & 4 & IUE (4) \\
CiU & 937.835 & 4,51 & 3 & LDR (2) \\
PPE (1) & & & & \\
COALICIÓN EUROPEA & 677.048 & 3,25 & 2 & LDR (1) \\
& & & & V-ALE (1) \\
C. NACIONALISTA + & & & & \\
EUROPA DE LOS PUEBLOS & 613.978 & 2,95 & 2 & V-ALE (2) \\
BNG & 349.079 & 1,68 & 1 & V-ALE (1) \\
EH & 306.920 & 1,48 & 1 & NI (1) \\
\hline
\end{tabular}


1994

\begin{tabular}{crrc}
\hline Escaños 94 & $\% 94$ & $N^{\circ}$ Votos 94 & Grupo Político 94 \\
\hline 28 & 40,12 & 7.453 .900 & PP \\
22 & 30,79 & 5.719 .707 & PSOE \\
9 & 13,44 & 2.497 .671 & IU \\
3 & 4,66 & 865.913 & CiU \\
2 & 2,79 & 518.532 & CC $\left(^{*}\right)$ \\
2 & 2,79 & 518.532 & UV $\left(^{*}\right)$ \\
2 & 2,79 & 518.532 & PAR $\left(^{*}\right)$ \\
0 & 0,76 & 140.445 & C. PODER ANDALUZ $\left(^{*}\right)$ \\
2 & 2,79 & 518.532 & C. NACIONALISTA $\left(^{*}\right)$ \\
0 & 1,29 & 239.339 & EUROPA DE LOS PUEBLOS $\left(^{*}\right)$ \\
0 & 0,75 & 139.221 & BNG \\
0 & 0,97 & 180.324 & HB \\
0 & 0,23 & 42.237 & ELS VERDS \\
\hline
\end{tabular}

TOTAL DE LOS PAÍSES DE LA UNIÓN EUROPEA

\begin{tabular}{lcc}
\hline & 1999 & 1994 \\
\hline Censo & 287.634 .673 & 265.741 .990 \\
Total Votantes & 142.085 .407 & 156.522 .032 \\
Participación & $49,4 \%$ & $58,9 \%$ \\
\hline
\end{tabular}

COMPOSICIÓN DEL PARLAMENTO EUROPEO

\begin{tabular}{lrr}
\hline & 1999 & 1994 \\
\hline PSE & 180 & 214 \\
PPE & 225 & 201 \\
ELDR & 43 & 42 \\
UPE & 17 & 36 \\
IUE-IVN & 35 & 34 \\
V & 37 & 27 \\
CRE & 14 & 21 \\
IEDN & 21 & 15 \\
NI & 18 & 37 \\
Otros & 36 & \\
TOTAL & 626 & \\
\hline
\end{tabular}




\section{GRUPOS POLITICOS}

- PPE: Grupo del Partido Popular Europeo (Demócrata-Cristianos) y de los Demócratas Europeos.

- PSE: Grupo del Partido de los Socialistas Europeos.

- ELDR: Grupo del Partido Europeo de los Liberales, Demócratas y Reformistas.

- V-ALE: Grupo de los Verdes en el Parlamento Europeo- Alianza Libre Europea.

- IUE-IVN: Grupo Confederal de la Izquierda Unitaria Europea - Izquierda Verde Nórdica.

- UEN: Unión por una Europa de las Naciones.

- EDD: Europa de las Democracias y de las Diferencias.

- NI: No Incrito.

\section{ANÁLISIS DE LOS RESULTADOS}

En las elecciones europeas celebradas el pasado 13 de junio de 1999, el centro-derecha consigue un magnífico resultado en relación con las elecciones europeas de 1994, propiciado fundamentalmente por el avance de los conservadores británicos y de los democristianos alemanes. Las formaciones del Partido Popular Europeo PPE han sido las más votadas en nueve de los quince países y este resultado convierte al PPE en el principal grupo de la Eurocámara con 225 diputados seguido por el Partido de los Socialistas Europeos con 180 escaños. Por otra parte, mantienen una importante presencia los liberales con 42 escaños y suben significativamente los Verdes (que pasan a tener 36 escaños frente a los 27 de 1994).

Asimismo, hay que destacar el bajo índice de participación global (el $23 \%$ en el caso de los británicos o el $45 \%$ en Alemania, por citar dos ejemplos). En España, el índice de participación fue del 64,3 por ciento, con lo que supera en 4,5 puntos a las elecciones celebradas en 1994 , un nivel de participación sólo superado por las elecciones europeas de 1987 , con un 68,5 por ciento de votantes.

En relación con los resultados obtenidos en España, al igual que en las elecciones europeas de 1994, el Partido Popular continúa siendo la primera fuerza política en el Parlamento de Estrasburgo, si bien no logra mantener los mismos resultados pues pierde un escaño aunque 
el descenso en número de votos es muy bajo; en 1994 el PP consiguió el $40,1 \%$ de los votos y 28 escaños y en estas últimas elecciones 27 escaños y el $39,62 \%$ de los votos.

La segunda fuerza política sigue siendo el PSOE, que mejora claramente sus resultados y logra atraer a parte del electorado de Izquierda Unida obteniendo dos escaños más y avanzando casi cinco puntos; en las elecciones europeas de 1994 el PP aventajó en casi diez puntos (el $9,8 \%$ ) al PSOE y en 1999 el margen se ha reducido a cuatro puntos. En 1994 los socialistas lograron el $30,8 \%$ de los votos y 22 escaños, mientras que en esta ocasión han obtenido 25 eurodiputados y el $35,37 \%$ de los votos.

La coalición de Izquierda Unida continúa como la tercera fuerza española en el Parlamento Europeo en número de votos, aunque es la gran perdedora. Pierde a más de la mitad de sus votantes de 1994 y cinco escaños de los nueve obtenidos en 1994 por lo que se queda sólo con cuatro eurodiputados.

Los nacionalistas catalanes de $\mathrm{ClU}$ se mantienen como cuarta fuerza política y conservan tres escaños, si bien bajan levemente en número de votos con respecto a 1994 (un 4,49\% frente al 4,7\% de 1994).

La quinta fuerza política es Coalición Europea, integrada por cuatro partidos regionalistas -Coalición Canaria, Partido Andalucista, Unión Valenciana y Partido Aragonés- que logra dos escaños y el $3,05 \%$ de los votos.

La sexta candidatura más votada ha sido la Coalición Nacionalista-Europea de los Pueblos que agrupa al PNV, Eusko Alkartasuna, Esquerra Republicana de Catalunya, Unió Mallorquina y EV-Confederación Ecologista de Cataluña, que mantiene dos escaños y consigue una ligera subida en el número de votos (2,96\% en 1999 y 2,8 en 1994).

Por vez primera, obtiene un escaño el Bloque Nacionalista Galego, pasando del $0,7 \%$ de los votos de 1994 al $1,68 \%$ mientras que los sucesores de Herri Batasuna, Euskal Herritarrok, vuelven a obtener un representante mejorando su resultado anterior (pasan de un $0,97 \%$ a un $1,47 \%$ de votos) y, finalmente, con un 1,44 de los votos, se queda sin escaño la coalición de Los Verdes-Las Izquierdas de los Pueblos (integrada por la Chunta Aragonesista, Esquerda de Galicia, Iniciativa per Catalunya-Verds, Izquierda Andaluza y Los Verdes). 